\title{
UCRL-PROC-216343
}

LAW RENCE LIVERMORE N A T IO N A L LABORATORY

\section{Materials Response under extreme conditions}

B. A. Remington, K. T. Lorenz, S. Pollaine, J. M. McNaney

October 19, 2005

2005 APS SCCM-14th American Physical Society Topical Conference on Shock Compression of Condensed Matter Baltimore, MD, United States July 31, 2005 through August 5, 2005 
This document was prepared as an account of work sponsored by an agency of the United States Government. Neither the United States Government nor the University of California nor any of their employees, makes any warranty, express or implied, or assumes any legal liability or responsibility for the accuracy, completeness, or usefulness of any information, apparatus, product, or process disclosed, or represents that its use would not infringe privately owned rights. Reference herein to any specific commercial product, process, or service by trade name, trademark, manufacturer, or otherwise, does not necessarily constitute or imply its endorsement, recommendation, or favoring by the United States Government or the University of California. The views and opinions of authors expressed herein do not necessarily state or reflect those of the United States Government or the University of California, and shall not be used for advertising or product endorsement purposes. 


\title{
MATERIALS RESPONSE UNDER EXTREME CONDITIONS B.A. Remington, K.T. Lorenz, S. Pollaine, J.M. McNaney
}

Tues. Oct. 4, 2005, 11:00 pm

\begin{abstract}
Solid state experiments at extreme pressures, 10 -100 GPa (0.1 - 1 Mbar) and strain rates $\left(10^{6}-10^{8} \mathrm{~s}^{-1}\right)$ are being developed on high-energy laser facilities. The goal is an experimental capability to test constitutive models for high-pressure, solid-state strength for a variety of materials. Relevant constitutive models are discussed, and our progress in developing a quasiisentropic, ramped-pressure, shockless drive is given. Designs to test the constitutive models with experiments measuring perturbation growth due to the Rayleigh-Taylor instability in solid-state samples are presented.
\end{abstract}

\section{INTRODUCTION}

Over the past decade, there has been a surge of activity in the field of materials science at extreme conditions of pressure, compression, and strain rate $\left(\mathrm{P}, \rho / \rho_{0}\right.$, and $\mathrm{d} \varepsilon / \mathrm{dt}$, resp.). This work is being done on high energy density (HED) facilities, such as high energy lasers, magnetic pinch facilities, and gas guns. [Remington, 2004a; 2005b; Reisman, 2001; Hayes, 2004; Huang, 2005] One of the long range goals for our work in this area, aimed at the National Ignition Facility (NIF) laser, [Hogan, 2001] is to experimentally test models of high pressure, high strain rate material strength, at pressures $\mathrm{P}>>100 \mathrm{GPa}(1 \mathrm{Mbar})$. In this article, we review aspects of our progress towards achieving this challenging goal.

\section{RESULTS AND DISCUSSION}

We begin with a discussion of constitutive models for material strength at high pressures and strain rates. The first model we mention is the Steinberg-Guinan (S-G) model, [Steinberg, 1980] which is essentially a first order Taylor expansion in pressure and temperature,

$$
\begin{gathered}
\sigma=\sigma_{0} f(\varepsilon)\left[1+\left(\frac{G_{P}^{\prime}}{G_{0}}\right) \frac{P}{\eta^{1 / 3}}+\left(\frac{G_{T}^{\prime}}{G_{0}}\right)(T-300)\right] \\
f(\varepsilon)=\left[1+\beta\left(\varepsilon_{i}+\varepsilon\right)\right]^{n}
\end{gathered}
$$

Here $\sigma_{0}, \varepsilon$, and $f(\varepsilon)$, and $\eta$ represent the ambient strength, strain, work hardening factor, and compression. The S-G model applies only to high strain rates, $\mathrm{d} \varepsilon / \mathrm{dt}>\sim 10^{5} \mathrm{~s}^{-1}$, and assumes that strength is independent of strain rate.
We next describe the Steinberg-Lund (S-L) model, which can be written as [Steinberg, 1989; Remington, 2004a]:

$$
\begin{aligned}
& \sigma=\left[\sigma_{T}+\sigma_{A} f(\varepsilon)\right] \frac{G}{G_{0}}, \\
& \frac{\sigma}{G}=\frac{\sigma_{T}}{G_{0}}+\frac{\sigma_{A}}{G_{0}} f(\varepsilon), \\
& \hat{\sigma} \approx \hat{\sigma}_{T}+\hat{\sigma}_{A} f(\varepsilon),
\end{aligned}
$$

where $\sigma, \sigma_{\mathrm{T}}, \sigma_{\mathrm{A}}$, and $\mathrm{f}(\varepsilon)$ are yield strength, the thermal and athermal components, and the work hardening factor (Eq. 1b), resp. The carrot symbol in Eq. (2c) indicates that the stresses have been normalized by the shear modulus, G. Starting with Orowan's equation for strain rate, $\dot{\varepsilon}=\rho_{\mathrm{m}} \mathrm{b}_{\text {disloc }}$, where $\rho_{\mathrm{m}}$ and $\overline{\mathrm{v}}_{\text {disloc }}$ correspond to mobile dislocation density and average dislocation velocity, we can relate the thermal component of the yield stress, $\sigma_{\mathrm{T}}$, to the strain rate. The form we write down differs slightly from the nominal Hoge-Mukherjee (H-M) model [Hoge, 1977], and is given by

$$
\dot{\varepsilon}=\frac{\rho_{\mathrm{m}} \mathrm{b}^{2}}{\frac{1}{\alpha v_{\mathrm{De}}} \exp \left\{\frac{2 \mathrm{U}_{\mathrm{k}}}{\mathrm{kT}}\left[1-\left(\frac{\sigma_{\mathrm{T}}}{\sigma_{\mathrm{P}}}\right)^{\mathrm{p}}\right]^{\mathrm{q}}\right\}+\left(\frac{\mathrm{D}^{*}}{\sigma_{\mathrm{T}}}\right)^{\mathrm{m}}}
$$

where $\mathrm{p}$ and $\mathrm{q}$ represent barrier shape parameters, $m$ is the power of the phonon drag term, $\mathrm{D}^{*}$ is the phonon drag coefficient (dimensionally different from nominal S-L or H$M$ drag coefficient (D), except when $m=1$ ), and no artificial cap is imposed on $\sigma_{\mathrm{T}}$. We will refer to this model as $\mathrm{H}-\mathrm{M}^{*}$. The nominal form of $\mathrm{H}$ $\mathrm{M}$ is recovered if $\mathrm{p}=1, \mathrm{q}=2$, and $\mathrm{m}=1$. 
To consider just the thermal activation component of Eq. 3, we set $\mathrm{D}^{*}=0$. For simplicity in comparisons to the PTW model below, let $\mathrm{p}=\mathrm{q}=\mathrm{m}=1$. Equation 3 can then be inverted to isolate the thermal component of the normalized flow stress, $\hat{\sigma}_{\mathrm{T}}=\sigma_{\mathrm{T}} / \mathrm{G}$, namely

$$
\hat{\sigma}_{\mathrm{T}}=\frac{\sigma_{\mathrm{A}}}{\mathrm{G}_{0}}+\frac{\sigma_{\mathrm{P}}}{\mathrm{G}_{0}}-\frac{\sigma_{\mathrm{P}}}{\mathrm{G}_{0}} \frac{\mathrm{kT}}{2 \mathrm{U}_{\mathrm{k}}} \ln \left(\frac{\dot{\varepsilon}_{0}}{\dot{\varepsilon}}\right),
$$

where $\dot{\varepsilon}_{0}=\alpha v_{\text {De }} \rho_{\mathrm{m}} \mathrm{b}^{2}, 1 / \alpha=2 \mathrm{w}^{2} / \mathrm{La}$, with $\mathrm{w}$, $L$, and $a$ as defined in [Steinberg, 1989; Hoge, 1977].

We wish to compare Eq. 4 with the PTW model, [Preston, 2003] which we write here in a simplified form as,

$$
\hat{\tau}=\hat{\tau}_{\mathrm{s}}-\left(\hat{\tau}_{\mathrm{s}}-\hat{\tau}_{\mathrm{y}}\right) \exp \left[-\frac{\theta \varepsilon}{\hat{\tau}_{\mathrm{s}}-\hat{\tau}_{\mathrm{y}}}\right] .
$$

Here $\hat{\tau}, \hat{\tau}_{\mathrm{s},}$ and $\hat{\tau}_{\mathrm{y}}$ correspond to the normalized total flow stress, and the respective limits for high strain $\left(\hat{\tau}_{\mathrm{s}}\right)$ and low strain $\left(\hat{\tau}_{\mathrm{y}}\right)$. When $\varepsilon \sim 0$ (low strain, negligible work hardening), Eq. 5 reduces to $\hat{\tau} \approx \hat{\tau}_{\mathrm{y}}=\tau_{\mathrm{y}} / \mathrm{G}$, namely

$$
\hat{\tau}=\max \left\{\mathrm{y}_{0}-\left(\mathrm{y}_{0}-\mathrm{y}_{\infty}\right) \operatorname{erf}\left[\kappa \hat{\mathrm{T}} \ln \left(\frac{\gamma \dot{\xi}}{\dot{\varepsilon}}\right)\right], \mathrm{s}_{0}\left(\frac{\dot{\varepsilon}}{\gamma \dot{\xi}}\right)^{\beta}\right\}
$$

The parameters are defined in [Preston, 2003] Considering only the thermal activation component initially, and limiting the discussion to the linear region of the error function, we approximate scaled flow stress as

$$
\hat{\tau} \approx \mathrm{y}_{0}-1.13\left(\mathrm{y}_{0}-\mathrm{y}_{\infty}\right) \kappa \hat{\mathrm{T}} \ln \left(\frac{\gamma \dot{\xi}}{\dot{\varepsilon}}\right),
$$

where $\dot{\varepsilon}$, and $\dot{\xi}$ represent the equivalent plastic strain rate and reference strain rate, resp. By comparing Equations 4 and 7, the correspondence between like terms in these two constitutive models allows us to correlate the PTW "free parameters" $\gamma, \kappa, \mathrm{y}_{\infty}$, and $\mathrm{y}_{0}$ with physical quantities from the $\mathrm{H}-\mathrm{M}^{*}$ model, giving

$$
\begin{gathered}
\gamma \sim \rho_{\mathrm{m}} \mathrm{b}^{2}, \quad \kappa \sim \frac{1}{\mathrm{U}_{\mathrm{k}}}, \quad \mathrm{y}_{\infty} \sim \hat{\mathrm{\sigma}}_{\mathrm{A}}, \\
\mathrm{y}_{0} \sim \hat{\mathrm{\sigma}}_{\mathrm{A}}+\hat{\mathrm{\sigma}}_{\mathrm{P}}, \quad \mathrm{y}_{0}-\mathrm{y}_{\infty} \sim \hat{\mathrm{\sigma}}_{\mathrm{P}}
\end{gathered}
$$

The correlation $\gamma \sim \rho_{\mathrm{m}} \mathrm{b}^{2}$ in Eq. 8 is particularly interesting. If the mobile dislocation density, $\rho_{\mathrm{m}}$, depends on the dynamics of the system, as suggested by recent molecular dynamics (MD) simulations of shocked $\mathrm{Cu}$, [Bringa, 2005] then $\gamma$ may also depend on the dynamics. Specifically, $\gamma$ may be different for a shock loaded system compared to ramped-pressure (shockless) driven system. [Lorenz, 2005b] Wherever $\rho_{\mathrm{m}}$ is a dynamically varying quantity, so too would the parameter $\gamma$ vary. This possibility needs to be examined more thoroughly in future work. This discussion also applies to the S-L, H-M, and the modified H-M* (Eq. 3) models, which treat $\rho_{\mathrm{m}}$ as a material constant, when in fact it may be evolving dynamically. It has already been suggested that variations in $\rho_{\mathrm{m}}$ can have a large effect on flow stress or material strength. [Remington, 2004a; Bringa, 2005] We are faced with, it seems, developing a theoretical approach to follow the evolution of $\rho_{\mathrm{m}}(\mathrm{t})$. Possibly very large scale MD simulations, [Bringa, 2005] or the new technique of multiscale dislocation dynamics plasticity (MDDP) will be beneficial in this task. [Zbib, 2002]

We illustrate these models in Fig. 1, as a function of strain rate, for $\mathrm{Ta}$ at $\mathrm{P}=0.5 \mathrm{Mbar}$, $\mathrm{T}=500 \mathrm{~K}$, and $\varepsilon=0.1$. The dot-dashed curve labeled (1b) corresponds to the nominal S-L model (Eq. 2a, but with the S-L cap on $\sigma_{\mathrm{T}}$ when $\sigma_{\mathrm{T}}>\sigma_{\mathrm{P}}$ ) with its nominal input parameters for Ta. [Steinberg, 1989] The dot-dashed curve labeled (1a) corresponds to Eq. 3 with $\mathrm{p}=1, \mathrm{q}=2$, $\mathrm{m}=1$, again with nominal $\mathrm{Ta}$ input parameters from [Steinberg, 1989]. Curves (1a) and (1b) coincide in the thermal activation regime, for $\mathrm{d} \varepsilon / \mathrm{dt}<\sim 10^{5} \mathrm{~s}^{-1}$. Above this strain rate, however, the nominal S-L model transitions essentially to the Steinberg-Guinan model, (Eq. 1) which is strain rate independent, and given by curve (1b) for $\mathrm{d} \varepsilon / \mathrm{dt}>\sim 10^{6} \mathrm{~s}^{-1}$. The modified HogeMukherjee model (Eq. 3), however, allows the phonon drag component to fully activate, and the strength increases steeply with strain rate, $\sigma \sim$ $\mathrm{d} \varepsilon / \mathrm{dt}$ for $\mathrm{m}=1$. Note, at high strain rates, where phonon drag dominates flow stress, as shown by curve (1a) in Fig. 1, $\sigma_{\mathrm{T}}>\sigma_{\mathrm{A}} \mathrm{f}(\varepsilon)$ in Eq. 2a. But $\sigma_{\mathrm{A}} \mathrm{f}(\varepsilon)$ contains the initial athermal microstructure and work hardening factor. If phonon drag is the dominant effect, flow stress $\sigma \sim \sigma_{\mathrm{T}} \mathrm{G} / \mathrm{G}_{0}$ is independent of $\sigma_{\mathrm{A}} \mathrm{f}(\varepsilon)$, and the strength is predicted to be independent of the initial microstructure and work hardening. The solid curve labeled (3) in Fig. 1 corresponds to 
the PTW model, Eq. 6, with nominal input parameters for Ta. [Preseton, 2003] In the low strain rate regime, $\mathrm{d} \varepsilon / \mathrm{dt}<\sim 10^{5} \mathrm{~s}^{-1}$, PTW also agrees with the S-L model. This is not surprising, since both models were "calibrated" against the same Hopkinson bar data. With nominal input parameters for Ta, the PTW model transitions to phonon drag at a higher strain rate, $\sim 10^{8} \mathrm{~s}^{-1}$, due to the higher nominal value for attempt frequency assumed. The transition is to a power-law "nonlinear" phonon drag model, with a softer dependence on strain rate, $\sigma \sim$ $(\mathrm{d} \varepsilon / \mathrm{dt})^{1 / 4}$, based on overdriven shock data. The modified $\mathrm{H}-\mathrm{M}^{*}$ model (Eq. 3 with $\mathrm{m}=4$ ) is shown in Fig. 1 by curve (2a). Here, the reference strain rate, $\dot{\varepsilon}_{0}$, has been increased by $\sim 100 \mathrm{x}$ over the nominal value, the Peierls stress has been scaled by $\mathrm{G}(\mathrm{P}, \mathrm{T}) / \mathrm{G}_{0}$, and the phonon drag power law parameter has been set to $\mathrm{m}=4$. Under these settings, the modified H-M model is consistent with the PTW model over nearly the entire strain rate range. For illustration, curve (2b) shows an intermediate version of $\mathrm{S}-\mathrm{L}$, in which the reference strain rate has been increased by a factor of $\sim 40$ over nominal, phonon drag has been "turned off" $\left(D^{*}=0\right)$, and the Peierls stress has been scaled by $G(P, T) / G_{0}$, which raises the $\sigma_{\mathrm{T}}$ cap.

We consider the PTW model in more detail in Figs. 2 and 3. In Fig. 2 we show the PTW flow stress vs. strain rate for $\mathrm{Ta}$ at 0.5 Mbar pressure at $\mathrm{T}=500 \mathrm{~K}$ temperature, as the input parameter $\gamma$ is varied over 4 orders of magnitude. It seems reasonable to require flow stress, $\sigma$, at low strain rate $\left(<\sim 10^{1} \mathrm{~s}^{-1}\right)$ to remain fixed. Hence, as $\gamma$ is decreased, $\kappa$ must be increased, for flow stress at low strain rate to remain unchanged, as shown in Fig. 2. Decreasing $\gamma$ increases flow stress, since $\gamma \sim \rho_{\mathrm{m}}$, whereas increasing $\kappa$ implies lower activation energy, which decreases flow stress. Hence, increasing $\kappa$ offsets the effect of decreasing $\gamma$. At a given flow stress, the transition to phonon drag (dashed curves in Fig. 2) happens at lower strain rate as $\gamma$ is decreased.

In Fig. 3, we show flow stress from the PTW model for variations in strain. It is seen that increasing strain beyond $\sim 0.4$ has no further hardening effect for Ta at $0.5 \mathrm{Mbar}$ and $\mathrm{T}=500$ $\mathrm{K}$. Also note that strain hardening does not affect flow stress in the phonon drag regime. In this regime, the dislocations are assumed to be gliding above the barriers, such that work hardening due to the accumulation of microstructure has little effect on flow stress. As discussed regarding Eq. 2a and curve (1a) in Fig. 1, the H-M* model (Eq. 3) leads to a similar conclusion.

We next discuss the results of an experimental technique for generating a very high pressure, high strain rate "drive" to compress samples in the solid state. This technique has been experimentally demonstrated up to peak pressures of $200 \mathrm{GPa}(2 \mathrm{Mbar})$ at the Omega laser, as shown in Fig. 4. [Edwards, 2004; Lorenz 2005b] The target consists of a low Z, low density reservoir (typically solid density plastic) of nominal thickness $\sim 0.2 \mathrm{~mm}$, followed by a $\sim 0.3 \mathrm{~mm}$ vacuum gap, then an $\mathrm{Al}$ sample. A laser pulse of energy $0.2-2 \mathrm{~kJ}$ in a temporally square pulse shape of duration $3-4$ ns is used to drive a strong shock through the low-Z reservoir. When the shock reaches the back side (the side opposite where the laser was incident), the reservoir "explodes" (unloads) into vacuum as a gas of "ejecta". The pressure that is applied to the sample results from the increasing ram pressure, $\quad P_{\text {ram }}=\rho_{\text {ejecta }} v_{\text {ejecta }}^{2}$, which increases smoothly and monotonically in time as the reservoir unloads. This technique for generating shockless compression was modeled after the early work of Barnes using high explosives (HE) as the source of the shock in the reservoir. [Barnes, 1974; 1980] The measured velocity profiles can be back integrated to infer the applied pressure vs. time at the front surface of the Al sample, using a technique developed by Hayes. [Hayes, 2004] We show in Fig. 4 the results from five different experiments, varying mainly the laser intensity, leading to peak pressures spanning 15-200 GPa (0.15 - 2 Mbar). As the peak pressure increases, the pressure rise time decreases. Nevertheless, even at 2 Mbar, with a $\sim 3$ ns rise time, the sample is not shocked, at least over the first 10-20 $\mu \mathrm{m}$ of Al. [Lorenz, 2005b] Furthermore, radiation-hydrodynamics simulations show that on future facilities, such as the NIF laser, [Hogan, 2001] this technique should be able to drive samples in the solid state to much higher pressures, $\mathrm{P}>10^{3} \mathrm{GPa}(10$ Mbar). [Remington, 2005a]

Finally, we show in Fig. 5 the results of 2D simulations of the Rayleigh-Taylor (RT) instability, [Lorenz, 2005a; Remington, 2004b] for an quasi-isentropically driven RT experiment in Ta at $\mathrm{P}_{\max } \sim 2$ Mbar. This experiment was designed for the first bundle of the NIF laser, [Hogan, 2001]. The simulations assumed the SG strength model, and varied the initial strength parameter, $\mathrm{Y}_{0}$. There is considerable sensitivity 
in the predicted RT growth factors vs. time, due to the stabilizing influence of material strength. The greater the strength, the lower the predicted growth factors. Strength models at very high pressure and strain rate can therefore be tested with this type of experiment. Recent experiments on the Omega laser in $\mathrm{V}$ and $\mathrm{Al}$ at lower peak pressure corroborate this prediction. [Lorenz, 2005a]

\section{CONCLUSION}

In summary, the field of extreme materials science is gaining considerable interest, and new results are emerging at a fast pace. In this article, we have discussed several high strain rate strength models, and summarized results from a ramped, shockless drive developed, to allow high pressure regimes in the solid state to be accessed. Constitutive models can be tested with RT experiments using this ramped drive.

\section{ACKNOWLEDGEMENTS}

*This work was performed under the auspices of the U.S. Department of Energy by the UC, Lawrence Livermore National Laboratory under contract W-7405-ENG-48. We are grateful for many illuminating discussions with D. Preston.

\section{REFERENCES}

[Barnes, 1974] J.F. Barnes, P.J. Blewett, R.G. McQueen, K.A. Meyer, and D. Venable, J. App. Phys., 1974, Vol. 45, 727;

[Barnes, 1980] J.F. Barnes, D.H. Janney, R.K. London, K.A. Meyer, and D.H. Sharp, J. Appl. Phys., 1980, Vol. 51, 4678.

[Bringa, 2005] Bringa et al., Nature, 2005, submitted.

[Edwards, 2004] J Edwards, K T Lorenz, B A Remington, S Pollaine, J Colvin, D Braun, B F Lasinski, D Reisman, J McNaney, J A Greenough, R Wallace, H Louis, and D Kalantar, Phys. Rev. Lett., 2004, Vol. 92, p. 075002.

[Hayes, 2004] D. B. Hayes, C. A. Hall, J. R. Asay, and M. D. Knudson, J. Appl. Phys., 2004, Vol. 96, 5520.

[Hogan, 2001] W.J. Hogan, E.I. Moses, B.E. Warner, M.S. Sorem et al., Nuclear Fusion, 2001, Vol. 41, 567.
[Hoge, 1977] K.G. Hoge and A.K. Mukherjee, J. Mat. Sci., 1977, Vol. 12, 1666.

[Huang, 2005] H. Huang and J.R. Asay, J. Appl. Phys., 2005, Vol. 98, p. 033524.

[Lorenz, 2005a] K.T. Lorenz et al., Phys. Plasmas, 2005, 12, 056309.

[Lorenz, 2005b] K.T. Lorenz et al., Phys. Plasmas, 2005, to be submitted.

[Preston, 2003] D.L. Preston, D.L. Tonks, and D.C. Wallace, J. Appl. Phys., 2003, Vol. 93, p. 211.

[Reisman, 2001] D.B. Reisman et al., J. Appl. Physics, 2001, Vol. 89, 1625.

[Remington 2004a] B.A. Remington, G. Bazan, J. Belak, E. Bringa, M. Caturla, J.D. Colvin, M.J. Edwards, S.G. Glendinning, D. Ivanov, B. Kad, D.H. Kalantar, M. Kumar, B.F. Lasinski, K.T. Lorenz, J.M. McNaney, D.D. Meyerhofer, M.A. Meyers, S.M. Pollaine, D. Rowley, M. Schneider, J.S. Stölken, J.S. Wark, S.V. Weber, W.G. Wolfer, B. Yaakobi, and L. Zhigilei, Met. Mat. Trans. A, 2004, 35A, p. 2587.

[Remington, 2004b] B.A. Remington, R.M. Cavallo, M.J. Edwards, B.F. Lasinski, K.T. Lorenz, H.E. Lorenzana, J. McNaney, S.M. Pollaine, and D.P. Rowley, B. Yaakobi, proceedings of the Third International Conference on Inertial Fusion Sciences and Applications, IFSA-2003, p. 917 (American Nuclear Society, La Grange Park, IL, 2004).

[Remington, 2005a] B.A. Remington et al., Astrophys. Space Science, 2005, 298, 235.

[Remington, 2005b] B.A.Remington et al., submitted, MST (2005)

[Steinberg, 1989] D.J. Steinberg and C.M. Lund, J. Appl. Phys, 1989, Vol. 65, 1528.

[Steinberg, 1980] D.J. Steinberg, S.G. Cochran, and M.W. Guinan, J. Appl. Phys, 1980, Vol. 51, 1496.

[Zbib, 2002] H.M. Zbib and T. Diaz de la Rubia, Int. J. Plasticity, 2002, Vol. 18, 1133. 


\section{FIGURE CAPTIONS}

Figure 1. Constitutive models. Flow stress (kbar) versus $\log$ strain rate for a variety of models (see text for details) for Ta at $0.5 \mathrm{Mbar}$, $500 \mathrm{~K}$ temperature, and plastic strain of 0.1: (1a) Hoge-Mukherjee (H-M), (1b) Steinberg-Lund (S-L), (2a) modified H-M, (2b) modified S-L, turning off phonon drag, and scaling the Peierls barrier with the pressure dependent shear modulus, and (3) the PTW model. [Remington, 2005b]

Figure 2. Flow stress (kbar) versus log strain rate for the PTW model for Ta at 0.5 Mbar, $500 \mathrm{~K}$ temperature, and plastic strain of 0.1 , showing that by varying two parameters $(\gamma, \kappa)$, the critical strain rate at which the transition to phonon drag occurs can be modified (indicated by the red vertical arrows), while leaving the low strain rate strength unchanged.

Figure 3. Predicted flow stress from the PTW model, as plastic strain is varied for Ta at 0.5 Mbar, $500 \mathrm{~K}$ temperature.

Figure 4: Ramped drive. Pressure vs. time for five different experiments at Omega, showing the ramped drive for maximum pressures spanning 0.15 kbar to 2 Mbar. [Edwards, 2004; Lorenz, 2005b]

Figure 5: Growth factor vs time (ns). Predicted RT growth of preimposed sinusoidal ripple (wavelength $\lambda=50 \mu \mathrm{m}$, initial amplitude $\eta_{0}=$ $0.2 \mu \mathrm{m}$ ) showing sensitivity to high pressure strength for Ta at 2 Mbar peak pressure. Note the $\log$ scale. 


\section{PTW, H-M, S-L for Ta, 0.5 Mbar, 500 K, 0.1 strain}

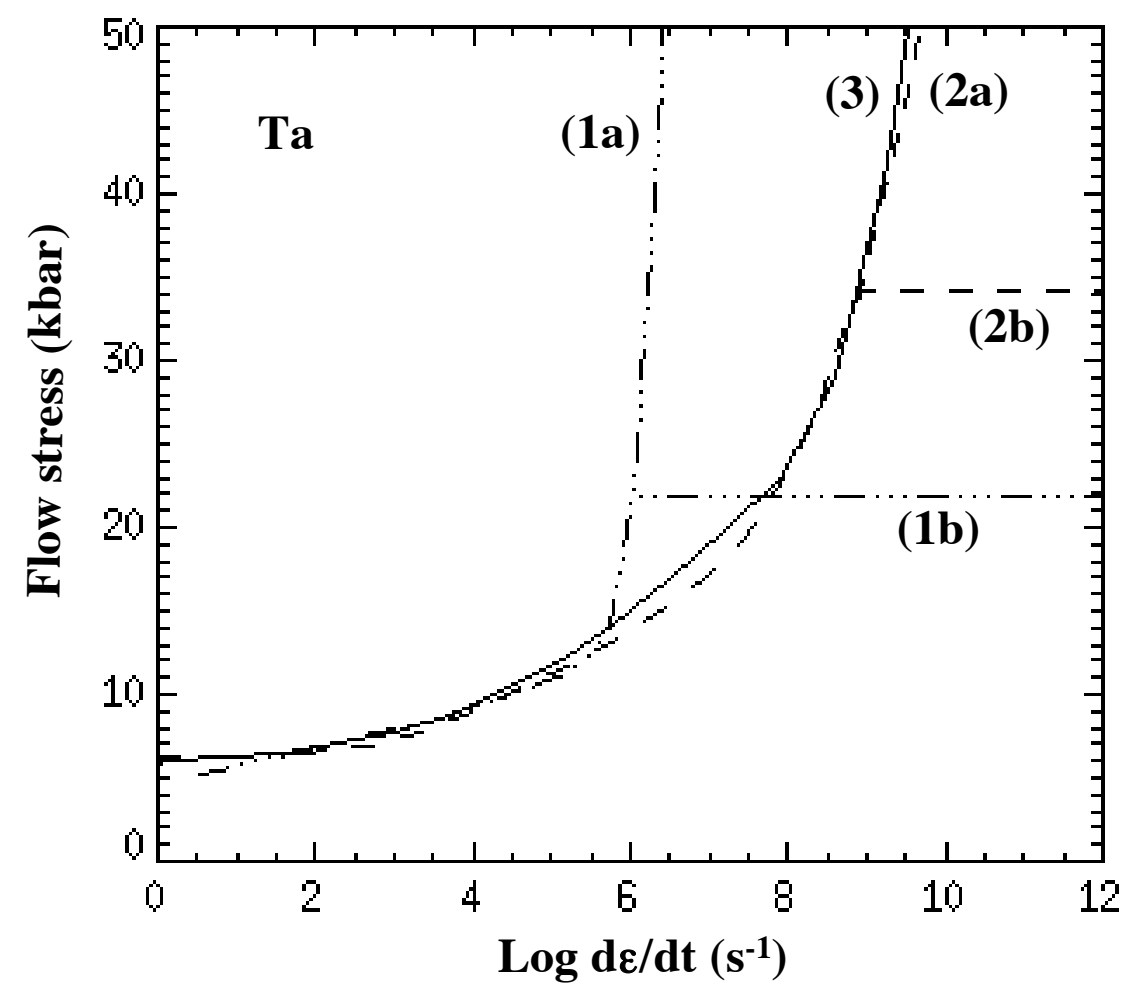

Figure 1 [Remington, submitted, MST (2005)]

.run PTW_2d2.pro (manilla PTW, Ta) (solid curve)

.run Steinberg-Lund_1f8.pro (various, Ta)

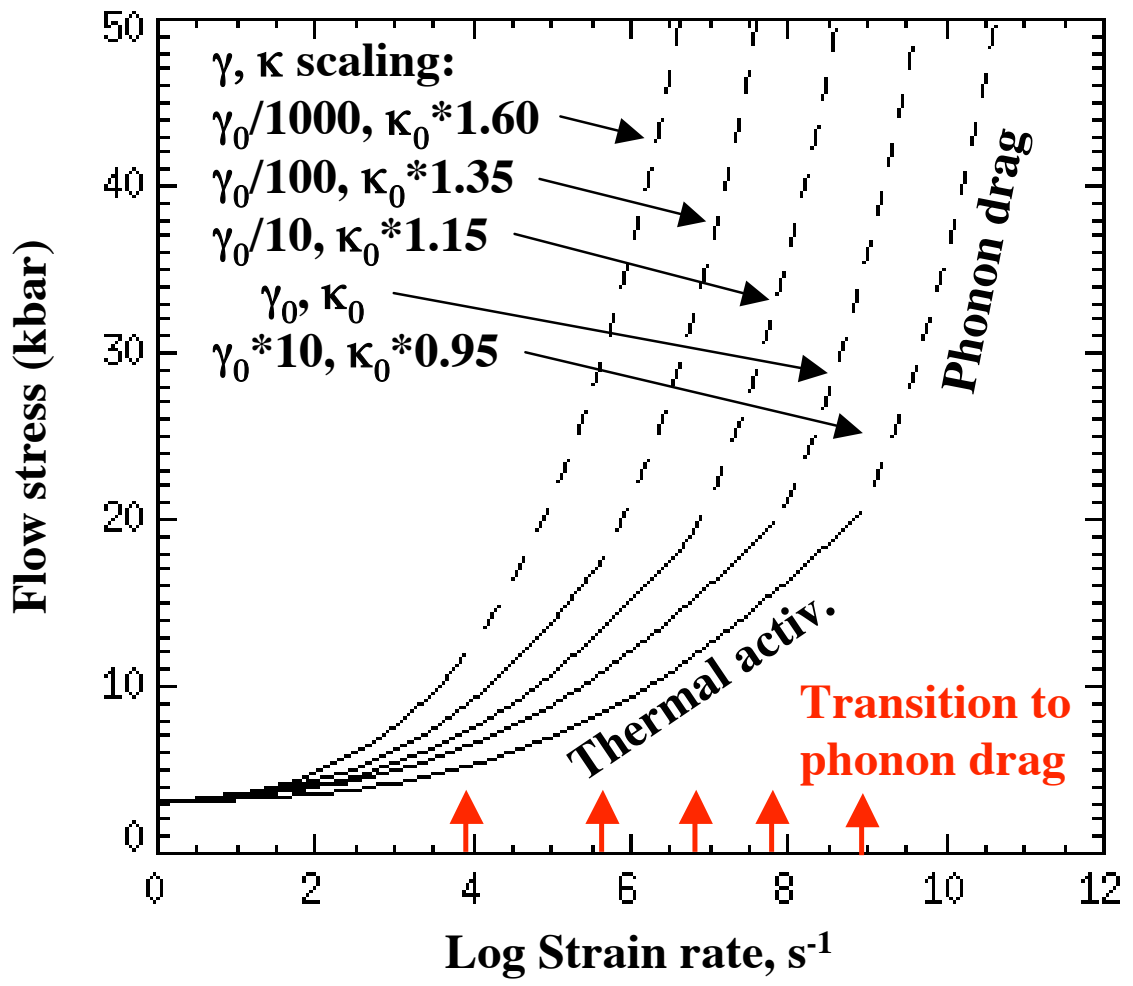

Figure 2

.run PTW_2d6.pro

Ta, $\mathrm{P}=0.5 \mathrm{Mbar}, \mathrm{T}=500 \mathrm{~K}$, strain $=0.1$ 


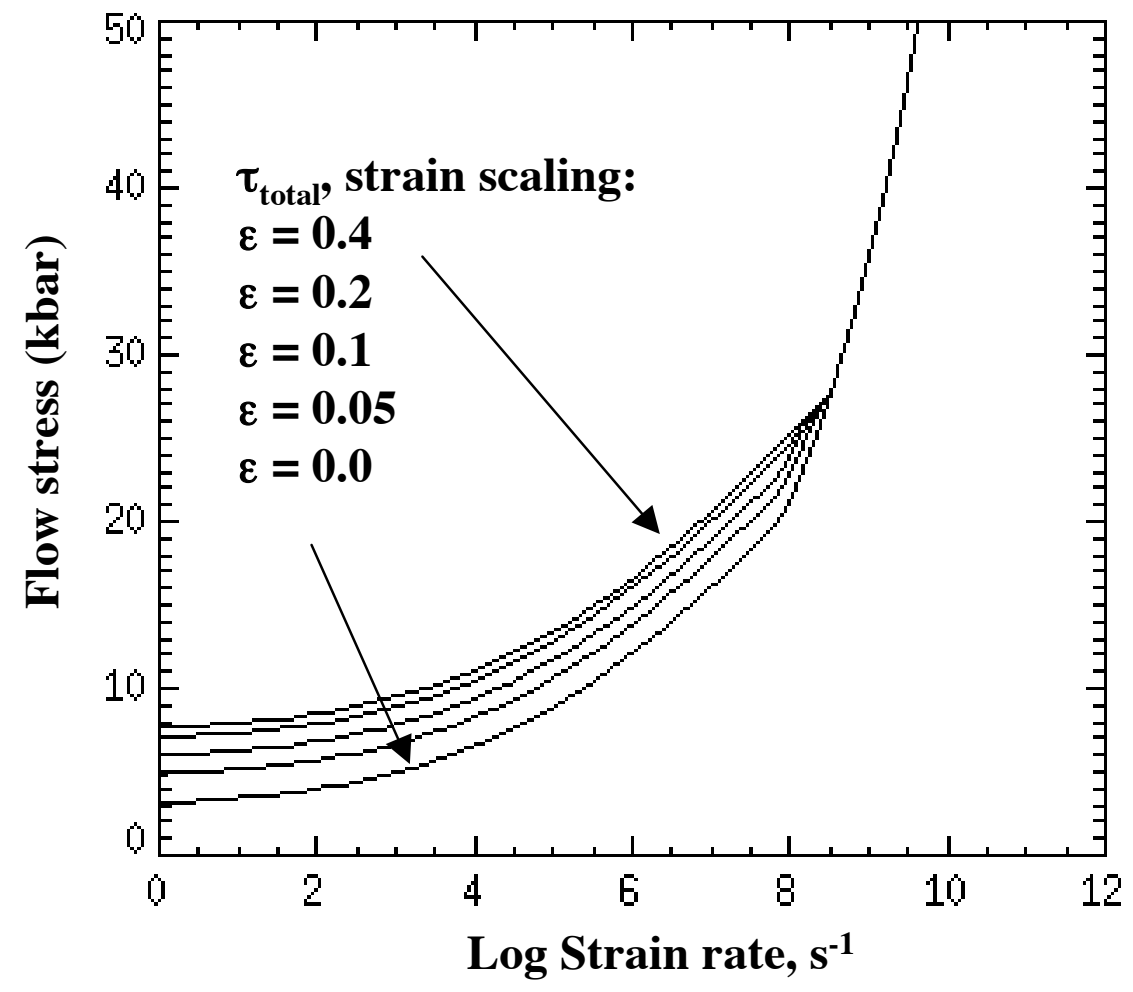

.run PTW_2d6.pro

Ta, $\mathrm{P}=0.5 \mathrm{Mbar}, \mathrm{T}=500 \mathrm{~K}$, strain scaling

Figure 3

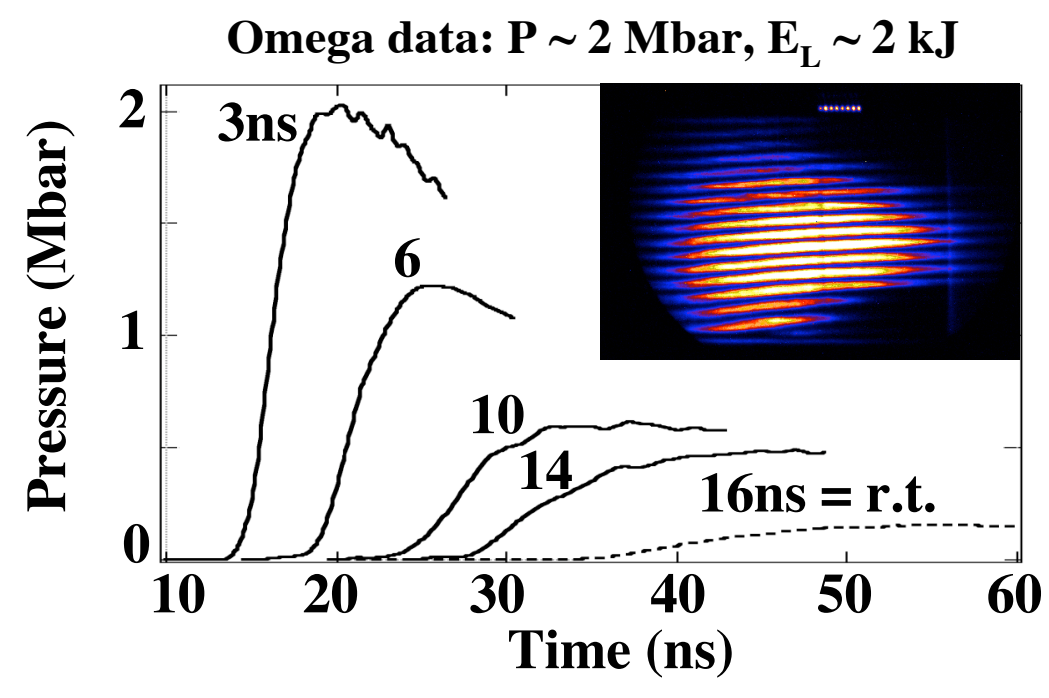

Figure 4

[Lorenz, JAP, to be submitted (2005)]

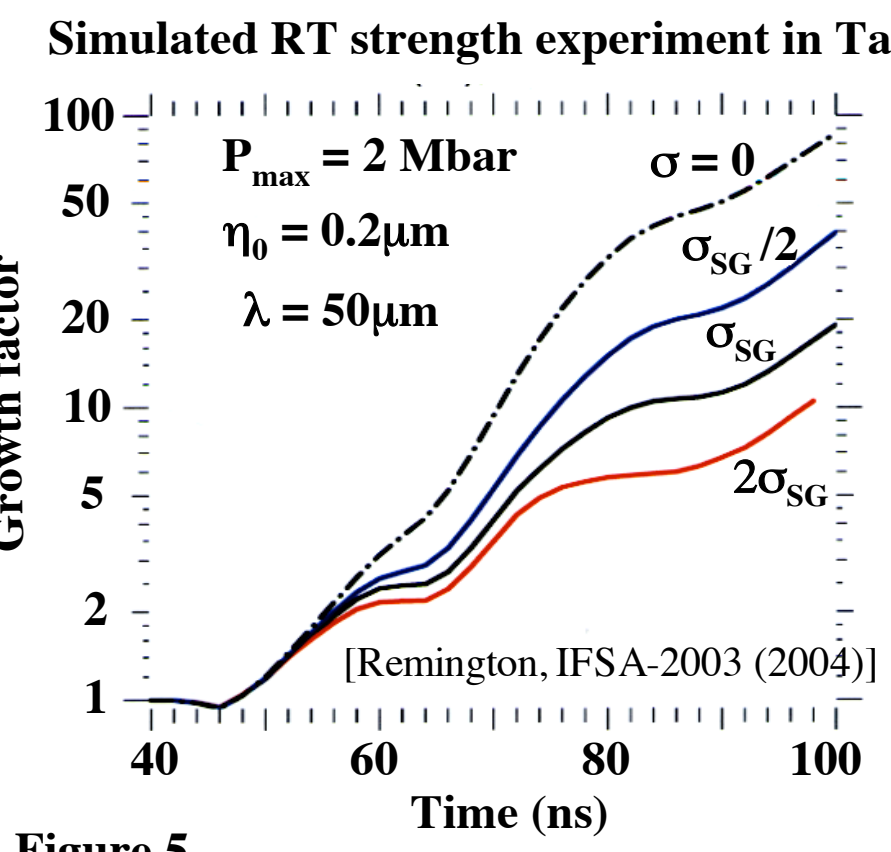

Figure 5

BAR_SCCM_figs_9.ppt;2 